\title{
Nested-Ring Mach-Zehnder Interferometer in Silicon-on-Insulator
}

\author{
S. Darmawan, Y. M. Landobasa, P. Dumon, R. Baets, and M. K. Chin
}

\begin{abstract}
For the first time, a nested-ring Mach-Zehnder interferometer (MZI) on silicon-on-insulator is realized using a complementary metal-oxide-semiconductor-based process. In this letter, we verify that the device operates in two modes: the inner-loop resonance dominant mode due to strong build-up inside the innerring, and the double-Fano resonances mode due to strong light interaction with the outer loop. The results show that the inner-loop resonance is highly sensitive to the MZI arm imbalance compared to the double-Fano resonance mode. Based on these considerations, we obtain a good fit between theory and experiment.
\end{abstract}

Index Terms-Guided waves, high-index contrast, integrated optic devices, Mach-Zehnder, microring resonators, nested ring, photonic wire, silicon-on-insulator (SOI) technology.

\section{INTRODUCTION}

$\mathbf{M}$ ICRORING resonators (RRs) are flexible building blocks in photonic integrated circuits for realizing various optical functionalities such as filters [1], [2], sensors [3], modulators [4], and switches [5]. In its basic configuration, the single ring resonator can be coupled to one or two bus waveguides. The fundamental design parameters are the ring cavity length $\left(L_{c}\right)$ and the coupling coefficient $(\kappa)$ between the ring and the bus. Of particular interest for sensing and switching applications is the microring-based Mach-Zehnder interferometer (MZI) structure where the RR is coupled to one arm of the MZI to give a resonant enhancement of the phase shift in that arm. By introducing a phase bias on the other arm of the MZI, asymmetric Fano resonances can be obtained that further enhances the sensitivity of this device. In an earlier paper [6], we have proposed a modified dual-bus RR (DBRR) structure, called the nested RR (NRR), and the resulting nested-ring MZI (NRMZI), that have interesting phase and transmission properties, in particular the box-like filter profile when certain conditions are satisfied. In this letter, we report for the first time the experimental realization of these devices fabricated in silicon-on-insulator (SOI) material, and show that they exhibit the various transmission profiles predicted by theory [6] but modified by the inevitable presence of MZI arm imbalance. In the next sections, we first review the theory of NRR and

Manuscript received July 2, 2007; revised August 30, 2007. The work of S. Darmawan was supported by the Singapore Millennium Foundation through a scholarship.

S. Darmawan, Y. M. Landobasa, and M. K. Chin are with the School of Electrical and Electronic Engineering, Nanyang Technological University, Singapore 639798, Singapore (e-mail: emkchin@ntu.edu.sg).

P. Dumon and R. Baets are with the Photonics Research Group, Department of Information Technology (INTEC), Ghent University-IMEC, 9000 Gent, Belgium (e-mail: Pieter.Dumon@intec.UGent.be).

Color versions of one or more of the figures in this letter are available online at http://ieeexplore.ieee.org.

Digital Object Identifier 10.1109/LPT.2007.910618
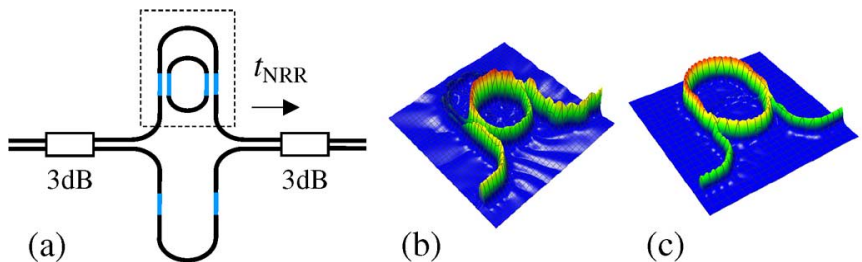

(b)

(c)

Fig. 1. (a) Schematic of NRMZI. The bare-MZI arm is designed to be balanced apart from the microring. The insets (b) and (c) show the FDTD simulations of the field distributions in the NRR when the inner- and outer-loop dominates, respectively. The notion $t_{\mathrm{NRR}}$ is the transmittivity of the NRR [6].

NRMZI, and then present the experimental data and their fit with theory.

\section{THEORY}

The schematic of NRMZI is shown in Fig. 1. The NRR is a ring nested by a U-shaped waveguide, and is equivalent to a dual-bus ring with the two buses connected by a loop with arbitrary length. Hence, the NRR may be considered as a DBRR loaded by the outer U-loop which behaves like a single-bus RR with respect to the inner DBRR. Topologically, the light has two possible paths to propagate, one in the inner ring, and one in the outer loop. Interference between these two possible paths can give rise to Fano resonances [7]-[13].

In the lossless limit, we expect the NRR to behave as an allpass filter with the phase response $\varphi_{\mathrm{NRR}}=\varphi_{t}+\varphi_{\text {load }}$, where $\varphi_{t}$ is the transmitted phase of the DBRR alone, and $\varphi_{\text {load }}$ is the effective phase introduced by the outer loop which is similar to the phase response of a single-bus RR. The significance of $\varphi_{\text {load }}$ depends on the coupling factor $\kappa^{2}$ between the bus and the ring. The field build-up in the outer loop is proportional to the transmissivity $|t|$ of the DBRR which increases with $\kappa^{2}$. When the outer loop is resonant $\varphi_{\text {load }}$ exhibits sharp changes of $2 \pi$. On the other hand, when $\kappa$ is small, the presence of the outer loop is felt less and the phase response is dominated by resonances in the inner ring. These two resonances are illustrated by the finitedifference time-domain (FDTD) simulations shown in Fig. 1.

In the NRMZI, the outer loop resonance results in steeper phase nonlinearity and hence gives rise to the sharp asymmetric Fano resonances. The transmission profile thus resembles a bandpass filter with sharp edges but also the ripples associated with the Fano resonance. These results are summarized in Fig. 2 for the ideal case of a balanced MZI for three values of $\kappa$. The left column shows the field build-up factors in the outer(solid line) and the inner-loop (dashed line) [6]. One can see that the strong build up in the outer loop corresponds to the sharp Fano resonances that define the edges of the "passband." Reducing the value of $\kappa$ flattens the ripples but at the expense of the band edge roll-off, resulting in a more Lorentzian profile 

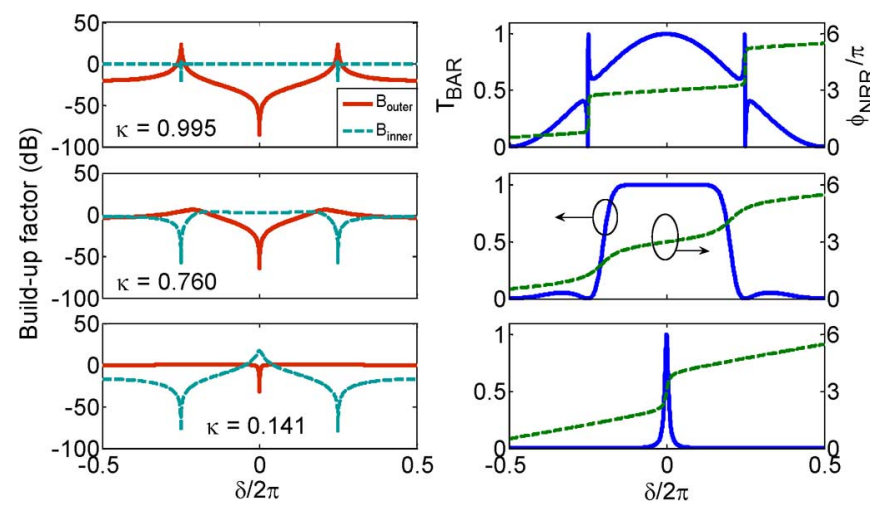

Fig. 2. (left) Build-up factor for the outer-loop $\left(B_{\text {outer }}\right)$ and the inner-loop ( $B_{\text {inner }}$ ) of NRR. (right) Corresponding $T_{\mathrm{BAR}}$ and $\varphi_{\mathrm{NRR}}$ as a function of normalized frequency, $\delta=2 \pi n_{g} L_{c} / \lambda$ assuming lossless case [6].

akin to the drop output of the DBRR when the light is resonant in the inner ring.

Aside from $\kappa$, another important parameter is the length $\left(L_{v}\right)$ of the outer loop, measured from center-to-center of the racetrack DC, as a ratio of the ring round-trip length. If this ratio, denoted as $v=L_{v} / L_{c}$, is an integer or half-integer, then the transmission spectrum will be periodic; otherwise it is chirped or random.

\section{EXPERIMENTAL RESULTS}

The NRMZI are fabricated in the Interuniversity Microelectronics Center (IMEC), under the ePIXnet silicon photonics platform using a complementary metal-oxide-semiconductor (CMOS)-based deep-ultraviolet (DUV) process [14]. The SOI consists of 220-nm silicon on a $2-\mu \mathrm{m}$ oxide buffer layer. In the DUV lithography process, the exposure dose is incrementally stepped up from the left to the right of the $8^{\prime \prime}$ wafer such that the gap width (waveguide width) increases (decreases) from left to right of the wafer. These variations imply that different devices on the wafer will have different coupling coefficients, and hence one can study the behavior of the NRMZI with various $\kappa$ values of interest. The UV resist is then used as the etch mask for an ICP etch through the Si layer to form the photonic wire waveguides. The typical loss of a $500-\mathrm{nm}$ width waveguide is $\sim 3 \mathrm{~dB} / \mathrm{cm}$ [14]. To facilitate fiber input and output coupling, each device is fabricated with vertical grating couplers [15] on a $10-\mu \mathrm{m}$-wide waveguide with an etch depth of $70 \mathrm{~nm}$ giving a second-order peak efficiency of about $20 \%$ at $1550 \mathrm{~nm}$. The $10-\mu \mathrm{m}$ waveguides are tapered down to the single-mode waveguides with nominal width of $475 \mathrm{~nm}$. The 3-dB couplers in the MZI are based on multimode interferometers (MMIs) with a width of $3.5 \mu \mathrm{m}$ and a length of $43.5 \mu \mathrm{m}$, with 5- $\mu \mathrm{m}$ taper length at the MMI input-output ports to minimize insertion loss. For the NRR, both point-coupled and race-track resonators are used. The microring radius is $15 \mu \mathrm{m}$.

The device transmission is measured with a broadband amplified spontaneous emission light source (1.41-1.62 $\mu \mathrm{m}$ ) and an optical spectrum analyzer. The input and output fibers are buttcoupled to the grating couplers at $10^{\circ}$ from vertical. Since the gratings are not optimized and couple only the TE polarization, while the light source is unpolarized, the total fiber-to-fiber insertion loss is found to be about $\sim 30 \mathrm{~dB}$. Fig. 3 shows the Drop
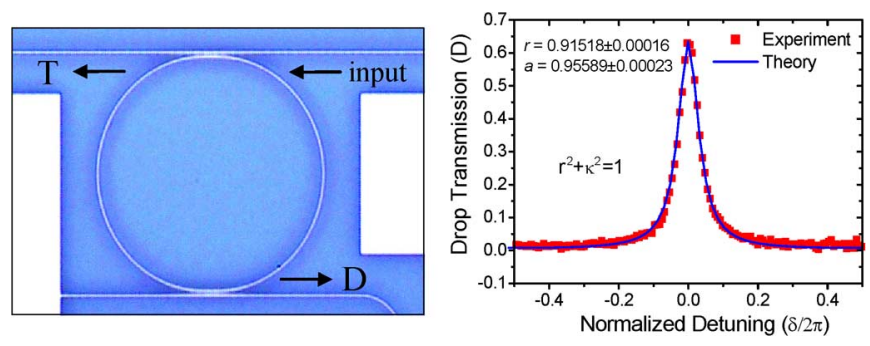

Fig. 3. (left) Fabricated DBRR with racetrack DC of $2 \mu \mathrm{m}$ and radius of $15 \mu \mathrm{m}$. (right) Experimental (with marker) and the theoretical fit (in solid line) of the DBRR drop $(D)$ transmission.

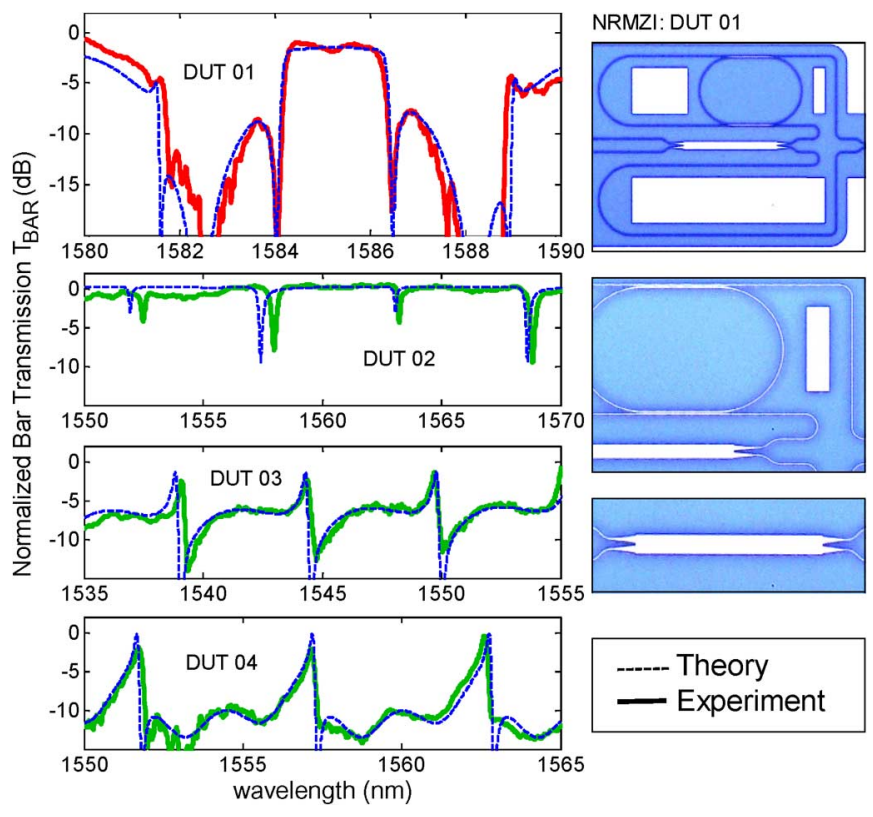

Fig. 4. Experimental results (solid lines) and theoretical fit (dashed lines) for DUT 01-04 (device under test). The right schematics show various parts of the fabricated DUT 01. The fit parameters are shown in Table I.

output of a conventional DBRR with a 2- $\mu \mathrm{m}$-long race-track and a radius of $15 \mu \mathrm{m}$ also fabricated on the same wafer. The drop spectra for the DBRR can be curve-fitted with the analytical formula $D=a \kappa^{4} /\left(1-2 a r^{2} \cos \delta+a^{2} r^{4}\right)$ [11], to give an independent derivation of $a$, where $a$ is the round-trip amplitude factor $a=\exp \left(-\alpha L_{c} / 2\right), \alpha$ is the loss coefficient, $\delta$ is the round-trip phase (or normalized frequency), and $r^{2}=1-\kappa^{2}$ is the self-coupling factor. The best fit value for $a$ thus obtained is 0.956 .

For the NRMZI, four devices were investigated: The first device (DUT 01) has a measured DC length of $17.58 \mu \mathrm{m}$ giving a relatively large calculated $\kappa$ value of 0.89 . The other three devices (DUT 02-04) consist of a point-coupled NRR with very small $\kappa$, with varying length of the outer loop corresponding to the nominal values of $v=1,1.5$, and 2.5, respectively. Fig. 4 gives the measured bar transmission spectra for these four devices, along with the theoretical fits that show good agreement with the data. The fit parameters are summarized in Table I.

Theoretical expressions for the spectra depend primarily on the parameters $a, \kappa$, and $\gamma$, where $\gamma$ is the ratio between the lengths of the lower and the upper bare MZI arms, i.e., $\gamma=1$ for balanced MZI arms. Therefore, $\Delta \gamma=\gamma-1$ represents an imbalance between the two MZI arms and is equivalent to a phase 
TABLE I

FIT PARAMETERS FOR DUT 01-04: ISOlATED WAVEGUIDE WIDTHS, DC WIDTHS, DC GAPS ARE 490, 475, $130 \mathrm{~nm}(01)$; 498, 484, $119 \mathrm{~nm}$ $(02,03) ; 483,468,140 \mathrm{~nm}(04)$, RESPECTIVELY. VALUES OF $v$ ARE MEASURED. THE $\Delta \varphi_{\text {offset }}$ IS CALCULATED AROUND $\lambda_{0} \approx 1.55 \mu \mathrm{m}$ WITH GROUP INDEX OF $n_{g} \sim 4.6$ [14]

\begin{tabular}{ccccccc}
\hline DUT & $\kappa$ & $a$ & $v$ & $\Delta \gamma=\gamma-1$ & $\begin{array}{c}\Delta \varphi_{\text {offset }} \approx \\
v \Delta \gamma \delta_{0}\end{array}$ & $n_{g}$ \\
\hline \hline 01 & 0.89 & 0.97 & 1.2287 & $+3 \times 10^{-5}$ & $+0.0284 \pi$ & 4.6062 \\
02 & 0.24 & 0.95 & 1.0006 & $-2 \times 10^{-3}$ & $-1.1260 \pi$ & 4.6275 \\
03 & 0.24 & 0.98 & 1.5006 & $-2 \times 10^{-3}$ & $-1.6866 \pi$ & 4.6210 \\
04 & 0.15 & 0.95 & 2.4991 & $+4 \times 10^{-5}$ & $+0.00564 \pi$ & 4.6428 \\
\hline
\end{tabular}

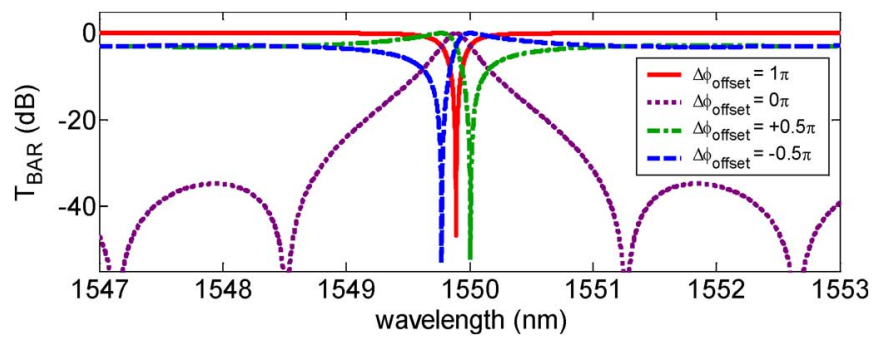

Fig. 5. Ideal theoretical plot of the bar transmission of the NRMZI $T_{\mathrm{BAR}}=$ $\sin ^{2}\left(\left[\varphi_{\mathrm{NRR}}-\left(v \delta+\Delta \varphi_{\mathrm{offset}}\right)\right] / 2\right)$ for the case of $v=1.5$ (DUT 03) with various effective phase biases at the lower MZI arm, assuming lossless case.

offset in the lower arm $\Delta \varphi_{\text {offset }}$. As expected from theory, DUT 01 , which has a relatively large $\kappa$, exhibits the double Fano resonance feature, giving a fairly flat-top response $(\sim 2$-nm bandwidth) and a sharp band roll-off $(\sim 7.5-\mathrm{dB}$ extinction ratio between the flat-top and the sidelobes). The best fit with theory is given by $a=0.97$ which is close to that given independently by Fig. 3, and by $\Delta \gamma=+3 \times 10^{-5}$ which is equivalent to a phase offset of $\Delta \varphi_{\text {offset }} \sim 0.0284 \pi$. A combination of design and fabrication errors resulted in the value $v=1.2287$, a significant deviation from the nominal integer or half-integer value, which partly accounts for the nonperiodicity and asymmetry in the transmission spectrum.

For the other three cases using point couplers where $\kappa$ is very small, the inner-loop of the NRR dominates, yet the output spectra can vary widely due to the random MZI arm imbalance. Similar to the case of microring-enhanced MZI, and as illustrated in Fig. 5, the bar transmission of the NRMZI can manifest three different signatures depending on the phase offset in the lower arm $\left(\Delta \varphi_{\text {offset }}\right)$. The output is 1$)$ similar to the Drop output of the DBRR (in the linear scale) when $\Delta \varphi_{\text {offset }} \sim 0$, as in DUT 04, 2) similar to the Through output of the DBRR when $\Delta \varphi_{\text {offset }} \sim \pm \pi$, as in DUT 02 , and 3 ) exhibits asymmetric Fano resonance when $\Delta \varphi_{\text {offset }} \sim \pm 0.5 \pi$, as in DUT 03. Similar spectral features have also been reported experimentally in [7]-[9]. The source of $\Delta \varphi_{\text {offset }}$ is due to fabrication variance and intrinsic phase imbalance. Even in perfectly fabricated MZI, the optical path in the upper MZI arm will deviate slightly from that in the lower arm due to the perturbation of the microring in the upper arm, inducing a slight phase offset between the two MZI arms.

In conclusion, for the first time, we have demonstrated NRMZI based on the SOI platform using a CMOS-based process, and obtained good agreement between theory and experiment. We show that the devices have two possible modes of operations depending on the ring-bus coupling coefficient $\kappa: 1)$ When $\kappa$ is small, the inner-loop resonance dominates leading to drop-like, through-like, or asymmetric Fano resonance output, depending on the offset phase arising from the MZI arm imbalance; 2) when $\kappa$ is relatively large, the outer loop resonance dominates giving rise to double-Fano resonances and a box-like transmission profile with sharp roll-off. For the small- $\kappa$ case, the microring has a higher $Q$, hence the device is more sensitive to slight MZI arm imbalance as compared to the device with larger $\kappa$ (lower $Q$ ). This sensitivity to offset phase implies that it may be desirable to have active bias control at the lower MZI arm (or in the NRR) in order to balance the MZI for better performance, or to tune the imbalance to achieve a variable output profile.

\section{REFERENCES}

[1] B. E. Little, S. Chu, J. Hryniewicz, and P. Absil, "Filter synthesis for periodically coupled microring resonators," Opt. Lett., vol. 25, pp. 344-346, Mar. 2000.

[2] S. Darmawan, Y. M. Landobasa, and M. K. Chin, "Pole-zero dynamics of high-order ring resonator filters," J. Lightw. Technol., vol. 25, no. 6, pp. 1568-1575, Jun. 2007.

[3] K. De Vos, I. Bartolozzi, E. Schacht, P. Bienstman, and R. Baets, "Silicon-on-insulator microring resonator for sensitive and label-free biosensing," Opt. Express, vol. 15, pp. 7610-7615, 2007.

[4] Q. Xu, S. Manipatruni, B. Schmidt, J. Shakya, and M. Lipson, "12.5 Gbit/s carrier-injection-based silicon micro-ring silicon modulators," Opt. Express, vol. 15, pp. 430-436, 2007.

[5] Q. Xu and M. Lipson, "All-optical logic based on silicon micro-ring resonators," Opt. Express, vol. 15, pp. 924-929, 2007.

[6] S. Darmawan, Y. M. Landobasa, and M. K. Chin, "Nested ring Mach-Zehnder interferometer," Opt. Express, vol. 15, pp. 437-448, 2007.

[7] J. E. Heebner, N. N. Lepeshkin, A. Schweinsberg, G. W. Wicks, R. W Boyd, R. Grover, and P.-T. Ho, "Enhanced linear and nonlinear optical phase response of AlGaAs microring resonators," Opt. Lett., vol. 29 , no. 7, pp. 769-771, 2004.

[8] P. P. Absil, J. V. Hryniewicz, B. E. Little, R. A. Wilson, L. G. Joneckis, and P. T. Ho, "Compact microring notch filters," IEEE Photon. Technol. Lett., vol. 12, no. 4, pp. 398-400, Apr. 2000.

[9] L. Zhou and A. W. Poon, "Fano resonance-based electrically reconfigurable add-drop filters in silicon microring resonator-coupled Mach-Zehnder interferometers," Opt. Lett., vol. 32, pp. 781-783, 2007.

[10] M. Kohtoku, S. Oku, Y. Kadota, Y. Shibata, and Y. Yoshikuni, "200-GHz FSR periodic multi/demultiplexer with flattened transmission and rejection band by using a Mach-Zehnder interferometer with a ring resonator," IEEE Photon. Technol. Lett., vol. 12, no. 9, pp. 1174-1176, Sep. 2000.

[11] L. Y. Mario, S. Darmawan, and M. K. Chin, "Asymmetric fano resonance and bistability for high extinction ratio, large modulation depth, and low power switching," Opt. Express, vol. 14, pp. 12770-12781, 2006.

[12] Y. Lu, J. Yao, X. Li, and P. Wang, "Tunable asymmetrical fano resonance and bistability in a microcavity-resonator-coupled Mach-Zehnder interferometer," Opt. Lett., vol. 30, pp. 3069-3071, 2005.

[13] Y. Lu, L. Xu, Y. Yu, P. Wang, and J. Yao, "Double-wavelength fano resonance and enhanced coupled-resonator-induced transparency in a double-microcavity resonator system," J. Opt. Soc. Amer., vol. 23, pp. 1718-1721, 2006.

[14] P. Dumon, W. Bogaerts, V. Wiaus, J. Wouters, S. Beckx, J. V. Campenhout, D. Taillaert, B. Luyssaert, P. Bientsman, D. V. Thourhout, and R. Baets, "Low-loss SOI photonic wires and ring resonators fabricated with deep UV lithography," IEEE Photon. Technol. Lett., vol. 16, no. 5, pp. 1328-1330, May 2004.

[15] D. Taillaert, P. Bienstman, and R. Baets, "Compact efficient broadband grating coupler for silicon-on-insulator waveguides," Opt. Lett., vol. 29 , no. 23 , pp. $2749-2751,2004$ 